\title{
Atomic String Potentials and the Form of [0001] Zone Axis Patterns of GaS, GaSe and InSe
}

\author{
John W. Steeds \\ Department of Physics, University of Bristol, Bristol BS8 1TL, UK
}

(Received March 1; accepted May 9, 1995)

\begin{abstract}
The relationship between the form of hexagonal zone axis patterns and the projected atomic potential is explored as a method for polytype identification of $\mathrm{GaS}, \mathrm{GaSe}$ and InSe. As the various known polytypes of these materials project to give unit cells with very different simple-string potentials this relationship may be exploited by making use of previous related work. It is found that this method is simple to apply and consistent with the results of more conventional analyses based on the symmetry of convergent beam electron diffraction patterns. Growth faults could, however, cause ambiguities in the method of analysis.
\end{abstract}

\section{Introduction}

It is interesting to review the [0001] large angle convergent beam electron diffraction (LACBED) zone axis patterns of De Blasi and co-workers published for $\mathrm{GaS}$, GaSe and InSe [1-4] in terms of the string potential concept. No doubt this is an activity that would have occupied his attentions in due course had circumstances been otherwise.

The idea of atomic string potentials is a natural consequence of the projection approximation that is often made in the case of diffraction of energetic particles by crystal lattices. It is widely used in channeling studies by high energy physicists [5] and it has proved extremely useful in high energy electron diffraction [6, 7] following on from the work of Berry [8], Buxton [9] and Bird [10]. One simply projects the crystal structure of interest along the direction of incidence. Lines of atoms aligned along zone axes produce cylindrically symmetric potential wells. Electron states in the crystal are either bound in a specific sub-set of wells or are free to wander from one well to another. Both wave and particle concepts may be used to analyse the electron diffraction that occurs (Berry [11]). This approach allows one to categorize diffraction problems according to the real-space arrangement and strength of atomic strings. In favorable cases it leads to straightforward analysis of the elaborate and often very beautiful forms of electron diffraction zone axis patterns. We here study the application of this approach to the published work of De Blasi and his co-workers. 


\section{The Projected Potentials of GaS, GaSe and InSe}

The form of zone axes that project with hexagonal symmetry and have projected potentials that can be formed from cylindrically symmetric atomic strings of various strengths has been quite thoroughly studied in the past $[12,13]$. According to the work of De Blasi et al. [1-4], and the references cited therein, the various common forms of $\mathrm{GaS}$, GaSe and InSe project into three versions of the three-string case, as illustrated in Figure 1. In the notation of De Blasi et al. [1-4] the $\beta-2 \mathrm{H}$ form, typical of $\mathrm{GaS}$, generates a projected potential that is composed of two identical atomic strings in the A position. The $\varepsilon-2 \mathrm{H}$ and $\delta-4 \mathrm{H}$ forms of GaSe project with a $(\mathrm{Ga}+\mathrm{Se})$ string in the A position, a Ga string in the B position and a Se string in the C position. The $\gamma-3 \mathrm{R}$ form of $\mathrm{GaSe}$ projects as a simple string case with identical $(\mathrm{Ga}+\mathrm{Se})$ strings in each of the $\mathrm{A}$, $\mathrm{B}$ and $\mathrm{C}$ positions. Frojected potentials of these types are similar to those formed by the various polytypes of transition metal chalcogenides and we will examine the zone axis patterns of De Blasi and co-workers in relation to the earlier work on these more widely studied compounds.

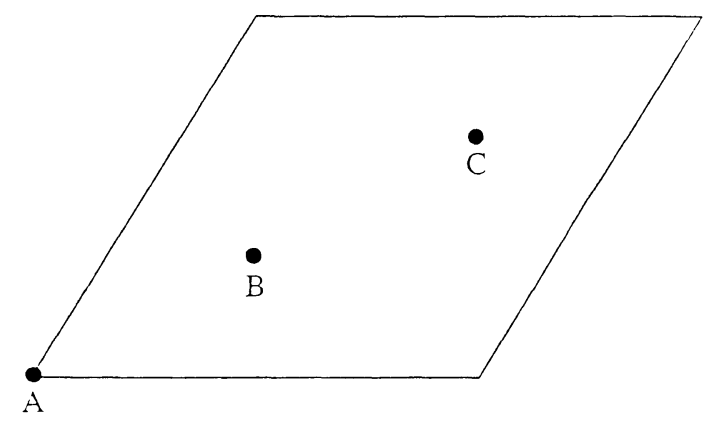

Fig. 1. - Schematic form of hexagonal projected potentials with three possible positions, A, B and C, for the location of atomic strings.

\section{Examination of Experimental Results}

$3.1 \beta-2 \mathrm{HGaS}-$ The [0001] projection of the $\beta-2 \mathrm{H}$ form of $\mathrm{GaS}$ gives rise to a projected potential of the same form as that of the $2 \mathrm{H}_{\mathrm{b}}$ polytypes $\mathrm{MoS}_{2}, \mathrm{MoSe}_{2}$ and $\mathrm{WSe}_{2}$. The strength of the atomic strings of $\mathrm{GaS}$ is given to a first approximation by $\frac{\left(Z_{\mathrm{Ga}}+Z_{\mathrm{S}}\right)}{c} \frac{a^{2}}{3 \sqrt{3}}$ while that of the transition metal (TM) chalcogenides $(\mathrm{C})$ is given by $\frac{\left(Z_{\mathrm{TM}}+2 Z_{\mathrm{c}}\right)}{c} \frac{a^{2}}{3 \sqrt{3}}$ [7]. In these expressions $Z_{\mathrm{A}}$ is the atomic number of the element $\mathrm{A}$, and $a$ and $c$ are the unit cell lattice constants. From these expressions we find that the string strength parameter for GaS (15.0) lies between that of $\mathrm{MoS}_{2}$ (11.6) and $\mathrm{MoSe}_{2}$ (17.7). It is therefore interesting to compare the results of De Blasi et al. with those of $\mathrm{MoS}_{2}$ and $\mathrm{MoSe}_{2}$, all recorded at a microscope operating voltage of $120 \mathrm{kV}$ (Fig. 2 ). In fact the details of these patterns have been shown to depend very strongly on the specimen thickness and the microscope operating voltage [14] so that an exact match would be very difficult to achieve. Nevertheless, the similarity of the three patterns in Figure 2 is evident and close study, 


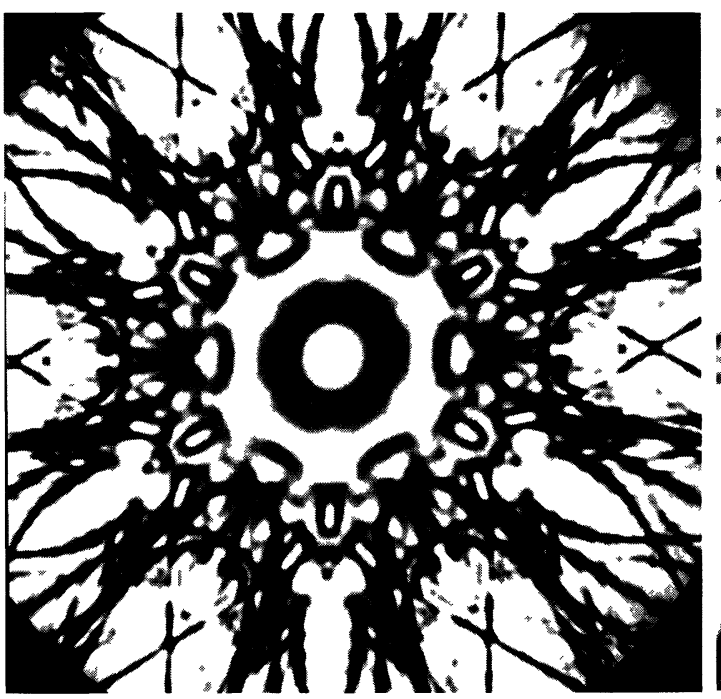

a)

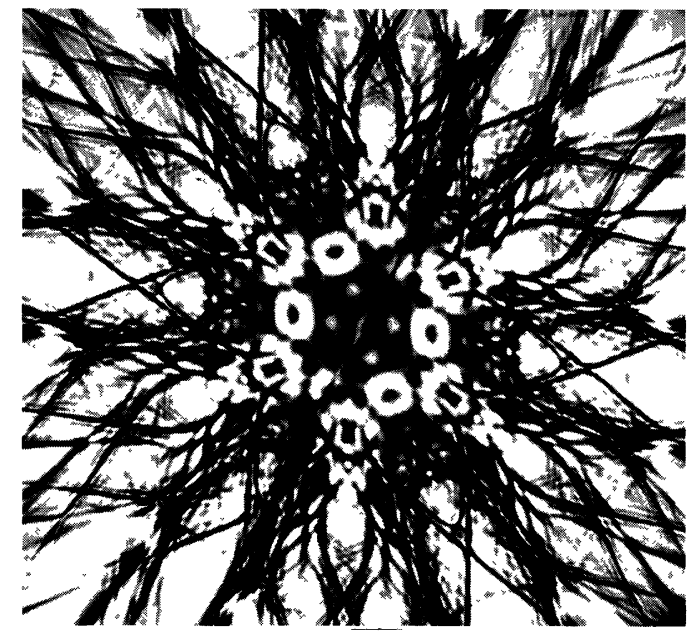

c)

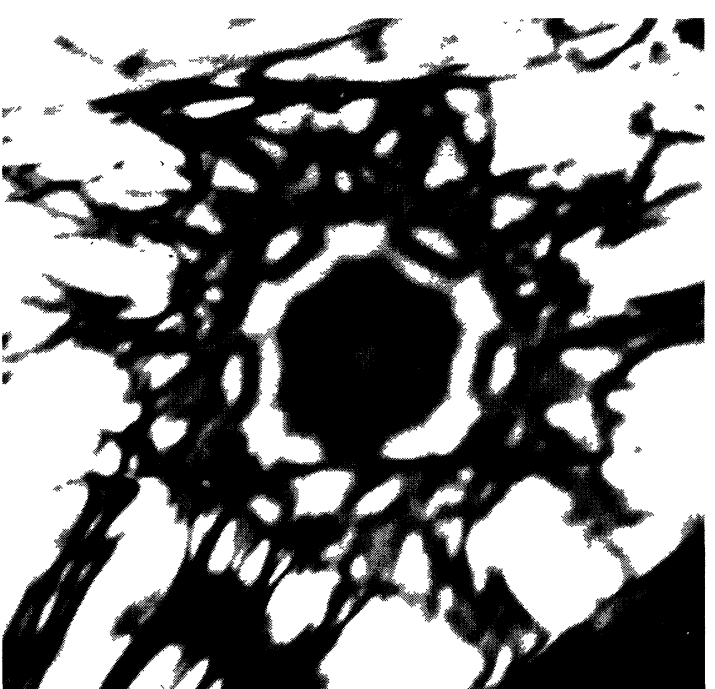

b)

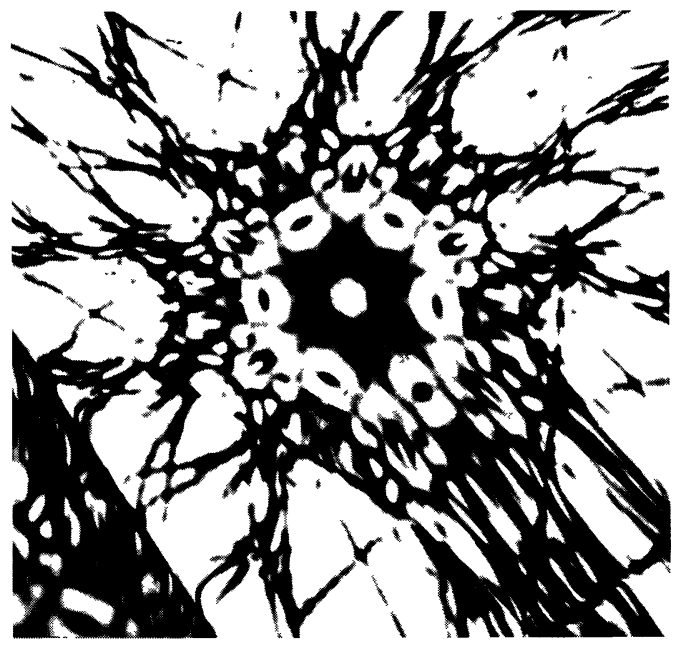

d)

Fig. 2. - a) [0001] LACBED pattern of $\beta-2 \mathrm{H} \mathrm{GaS}$ (Fig. 2c of [1]) to be compared with b) a [0001] bend contour pattern of $\mathrm{MoS}_{2} \mathrm{c}$ ) a further [0001] LACBED pattern of $\beta-2 \mathrm{H} \mathrm{GaS} \mathrm{(Fig.} 1$ of [3]) for a different film thickness (with a dislocation crossing the centre of the pattern) compared with d) a [0001] bend contour pattern of $\mathrm{MoS}_{2}$. All patterns were recorded at a microscope operating voltage of $120 \mathrm{kV}$.

involving patterns obtained at different specimen thicknesses and operating voltages, indicates that the GaS result is closer to those of $\mathrm{MoSe}_{2}$ than $\mathrm{MoS}_{2}$.

\subsection{R GaSe and InSe - According to the work of Steeds et al. [7] these simple-string zone axes} have string strengths given approximately by $\frac{2\left(Z_{\mathrm{Ga}}+Z_{\mathrm{Se}}\right)}{c} \frac{a^{2}}{3 \sqrt{3}} \approx 14.3$ and $\frac{2\left(Z_{\mathrm{In}}+Z_{\mathrm{Se}}\right)}{c} \frac{a^{2}}{3 \sqrt{3}} \simeq$ 20.5 
These values put the $3 \mathrm{R}$ GaSe zone axis between the $<111>$ zone axis of Si, which has a string strength of 12.7, and that of $\mathrm{Ag}<111>$ which has a string strength of 16.0. InSe lies between the values for $\mathrm{Ag}<111>$ and $\mathrm{Au}<111>$, with a string strength of 27.1, and $\mathrm{Ge}<111>$ with a string strength of 30.2. The patterns that they show (for example, Fig. 1 of [2]) are hence very similar to previously published results for simpler materials $[6,13]$ except those for $\mathrm{Si}<111>$ where the absorption is considerably lower so that ring patterns dominate the centre of the silicon patterns but are extinguished in the cases of GaSe and InSe and also for $\mathrm{Ag}, \mathrm{Ge}$ and $\mathrm{Au}<111>$ patterns.

$3.3 \varepsilon-2 \mathrm{H} \mathrm{GaSe}-$ This case is rather similar but somewhat different from others that have been studied previously [12] because each of the three strings is of different strength. However, since the atomic number difference between $\mathrm{Ga}$ and $\mathrm{Se}$ is rather small (31 and 34 respectively) we can expect strong similarities to the cases when the $\mathrm{B}$ and $\mathrm{C}$ string strengths are identical. The A strings, by way of comparison, are much stronger and depend on $\left(Z_{\mathrm{Ga}}+Z_{\mathrm{Se}}\right)$. As a result, we conclude that the projected potential is rather similar to that of $2 \mathrm{H} \mathrm{TaSe} \mathrm{T}_{2}$ in that the $\mathrm{A}$ atomic strings are approximately twice as strong as the $\mathrm{B}$ and $\mathrm{C}$ strings. This conclusion is consistent with a comparison (Fig. 3) between the published results for $\varepsilon-\mathrm{GaSe}$ and previously unpublished results for $2 \mathrm{H} \mathrm{TaSe}_{2}$ (but see [12] for other related zone axis patterns).

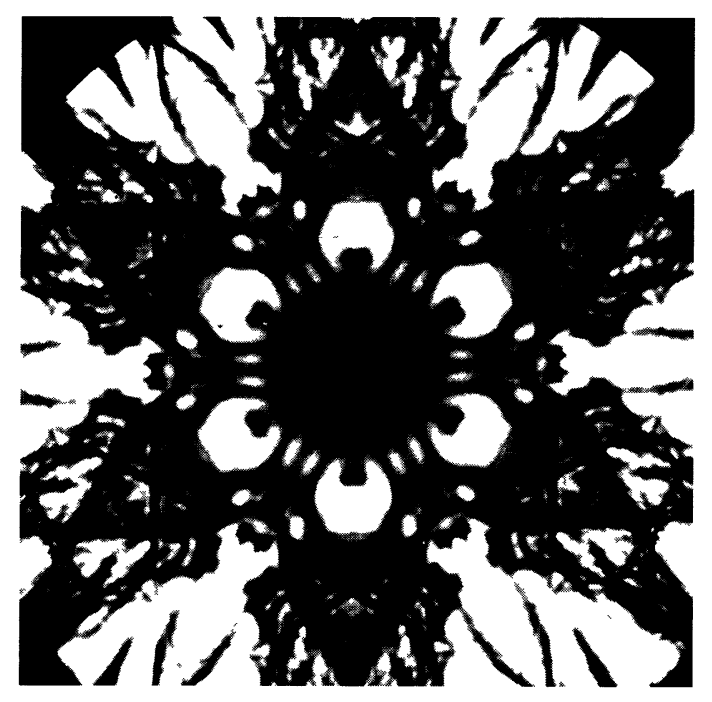

a)

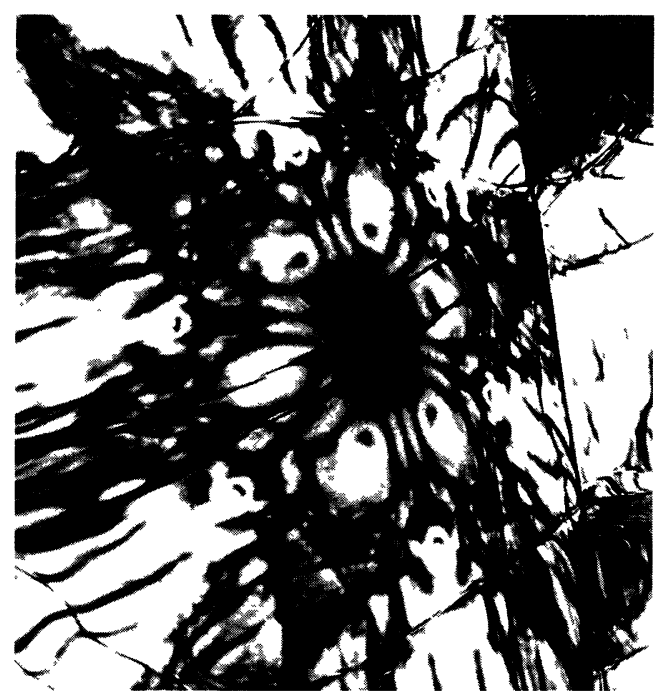

b)

Fig. 3. - a) [0001] LACBED pattern of $\varepsilon-2 \mathrm{H} \mathrm{GaSe}$ (Fig. 2a of [1]) compared with b) a [0001] bend contour patterns of $2 \mathrm{H} \mathrm{TaSe} 2$ both taken at $120 \mathrm{kV}$.

\section{Discussion}

The work of De Blasi [1-4] and co-workers in identifying polytypes of GaS, GaSe and InSe has been confirmed by a completely independent method of analyzing their results. The wealth of detail present in zone axis patterns and the eye's ability at pattern recognition makes it a relatively simple matter to compare experimental results obtained from unknown polytypes with previously 
published results. The classification of zone axis patterns with hexagonal symmetry follows a wellestablished route, depending on the number of independent atoms strings per projected unit cell and their strengths, defined here by a simple formula. It should however be pointed out that a more precise definition of atomic string strength is given elsewhere [7] and should be used in cases where detailed examination is required. In the case of the zone axis patterns studied here the differences were so great and the similarities to existing results in the literature so strong that the more accurate determination of string strengths was unnecessary.

Of course this method used for polytype identification has its limitations. As the results depend on projected structure, stacking disorder may not be immediately apparent and the patterns from disordered materials could give misleading results. For example, typical disorder could lead to slabs of ordered material displaced so that A positions in the projected unit cell are translated to $\mathrm{B}$ or $\mathrm{C}$ positions of adjoining slabs. Often when this occurs the result is a lowering of the zone axis pattern symmetry from $6 \mathrm{~mm}$ (typically to $3 \mathrm{~m}$ or m symmetry). However, such symmetry breaking may not be apparent without taking recourse to the more sensitive techniques of symmetry determination by convergent beam electron diffraction exploited by De Blasi and his associates.

However, zone axis patterns from defect free polytypes do have the advantage that they may be used to determine the nature of the projected charge distribution. Either by zone axis critical voltage measurements [12] or by the extension of methods currently in use on Si, Ge and diamond $[15,16]$ accurate structure factor information can be obtained by well worked-out procedures. The structure factors may then be used to determine the charge distributions. By use of energy filtering of the results and attention to a number of practical details, very accurate results are now being achieved this way.

\section{Summary and Conclusions}

It has been demonstrated that the atomic string potential concept can be applied to the problem of identification of the polytypes of $\mathrm{GaS}, \mathrm{GaSe}$ and InSe previously identified by other methods by De Blasi and his associates [1-4]. This method makes use of information present in large angle convergent beam electron diffraction results that are generated very simply in the course of investigation of materials by electron diffraction. It is limited to zone axes that project in the form of a relatively small number of independent atomic strings and to defect free regions of the material under study.

\section{Acknowledgements}

I would like to thank D. Manno for providing original copies of the micrographs reproduced here from her work with Prof. De Blasi.

\section{References}

[1] De Blasi C., Manno D., Rizzo A., "Convergent Beam Electron Diffraction Study of Melt and Vapour Grown Single Crystals of Gallium Chalcogenides”, Il Nuovo Cimento D 11 (1989) 1145-1163.

[2] De Blasi C., Manno D., Rizzo A., "Study of the Polytypism in Melt Growth InSe Single Crystals by CBED”, J. Cryst. Growth 100 (1990) 347-353. 
[3] De Rlasi C., Manno D. and Rizzo A., "Convergent Beam Electron Diffraction Characterization of Dislocations in GaS Single Crystals”, Ultramicrosc. 33 (1990) 143-140.

[4] De Blasi C., Mancini A.M., Manno D., Rizzo A. and Carlino E., "CBED Analysis of GaSe Crystals Grown from the Melt by Different Doping Elements", Il Nuovo Cimento D13 (1991) 233-246.

[5] For example, Thompson M.W., Contemp. Phys. 9 (1958) 375.

[6] Shannon M.D. and Steeds J.W., Philos. Mag. 36 (1977) 279.

[7] Steeds J.W., Jones P.M., Loveluck J.E., Cooke K., Philos. Mag. 36 (1977) 309.

[8] Berry M.V., J. Phys. C: Solid St. Phys. 4 (1971) 697.

[9] Buxton B.F. and Tremewan P.T., Acta Cryst. A36 (1980) 304.

[10] Bird D.M., J. Electron. Micros. Tech. 13 (1989) 77.

[11] Berry M.V., Buxton B.F., Ozorio de Almeida A.M., Radiat. Eff. 20 (1973) 1.

[12] Fung K.K. and Steeds J.W., Ultramicrosc. 31 (1989) 399.

[13] Steeds J.W. and Vincent R., J. Microsc. Spectrosc. Electron 8 (1983) 419.

[14] Jesson D.E. and Steeds J.W., Ultramicrosc. 31 (1989) 399.

[15] Bird D.M. and Saunders M.A., Ultramicrosc. 45 (1992) 241.

[16] Midgley P.A., Saunders M.A., Vincent R. and Steeds J.W., Ultramicrosc. 59 (1995) 1. 